\title{
Vulnerabilidad, alto riesgo y prevención de la esquizofrenia: una perspectiva cognitiva $\left(^{*}\right)$
}

\author{
JOSÉ MARÍA RUIZ-VARGAS \\ Universidad Autónoma de Madrid
}

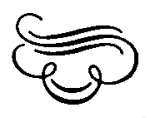

Resumen

Ante el becho sobradamente constatado de que el episodio esquizofrénico es profundamente desorganizador y su recuperación difícil e impredectible, se está prestando, en años recientes, una especial atención y esfuerzo al desarollo y puesta en práctica de programas preventivos. El modelo de vulnerabilidad a la esquizofrenia parece configurarse como el marco teórico y de trabajo más adecuado para tales programas. Tras revisar los postulados básicos de dicho modelo se presenta una revisión de los más relevantes estudios experimentales que están permitiendo la identificación de marcadores cognitivos de vulnerabilidad a la esquizofrenia en poblaciones de alto riesgo*. Se apunta, a continuación, la posibilidad de elaborar una batería que permita la identificación precoz de los sujetos que podrian convertirse en esquizofrénicos. Se analiza someramente algunos de los más importantes programas preventivos de esquizofrenia y se concluye que aún es pronto para emitir un juicio acerca de la eficacia de los mismos. Se deja patente, finalmente, que si bien todavía no estamos preparados para prevenir la esquizofrenia nos encontramos en el camino adecuado para conseguirlo.

\section{Abstract}

Because a schizophrenic episode is so disorganizing and recovery so arduous and unpredictable, attention has recently shifted to the feasibility of preventive programs. This paper presentas, first, the vulnerability model as a suitable theoretical framework for those programs, and basic assumptions of this model are reviewed. Second, a survey of the most relevant experimental studies for identification of cognitive markers of vulnerability to schizophrenia in *high-risk * samples is exposed. Third, the possibility of devicing a battery for early identificstion of subjects who could become schizophrenics is mentioned. Fourth, some preventive programs of shcizophrenia are briefly analyzed, and it is concluded that it is too soon to assess the possible efficiency of those programs. Finally, it is stressed that, even though we are still not ready to prevent schizphrenia, we are on a right road for that.

(*) El contenido de este trabajo es una versión modificada de la comunicación que, con el título de Vulnerabilidad y prevención de la esquizofrenia: El pradigma del alto riesgo, fue presentada por el autor en el III Congreso Nacional de Autismo y Psicosis Infantiles. Madrid, 24-27 de abril de 1985 .

Dirección del autor: José María Ruiz Vargas. Departamento de Psicología Básica, Social y Metodología. Facultad de Psicología. Universidad Autónoma de Madrid. Ciudad Universitaria de Canto Blanco. 28049 Madrid.

Artículo recibido durante el año 1985. 


\section{INTRODUCCION}

Es una opinión ampliamente difundida y aceptada en los ambientes científicos implicados, de una u otra forma, en la esquizofrenia que esta enfermedad es una de las formas más graves de desorden mental y que representa, por tanto, uno de los mayores problemas de salud mental de nuestro tiempo. Ello vendría determinado, en primer lugar, por su elevada incidencia (aproximadamente el $1 \%$ de la población) y, por otro lado, por los devastadores efectos que produce, tanto a nivel individual, como social.

$\mathrm{Si}$ bien en los últimos años diferentes estudios de seguimiento de grupos elevados de pacientes han coincidido en señalar - frente a las opiniones más tradicionales- la naturaleza más benigna de la esquizofrenia (e.g., M. Beuler, 1972; Schapiro y Shader, 1979; Ciompi, 1980, 1984; Huber, Gross, Schtuller y Linz, 1980), hasta el punto de hablarse de «la metamorfosis de la esquizofrenia» (Zubin, Magaziner y Steinhauer, 1983), sigue siendo un hecho fácilmente constatable que el episodio esquizofrénico es tremendamente desorganizador y su recuperación difícil e impredectible.

En consecuencia, a partir de las dos últimas décadas, se está prestando una especial atención y esfuerzo a las posibilidades de los programas preventivos.

Ahora bien, para prevenir necesitamos unos conocimientos acerca de la etiología del trastorno en cuestión, en este caso de la esquizofrenia. Si bien es cierto que la etiología de la esquizofrenia continúa envuelta en el misterio, no lo es menos el hecho de que contamos con diferentes teorías o modelos que están posibilitando la identificación de determinados signos o marcadores -independientes de la sintomatología esquizofrénica $y$, por tanto, anteriores a la misma- indicadores de un mayor riesgo en unos individuos que en otros.

Precisamente el concepto de vulnerabilidad a la esquizofrenia -incluido en el modelo de Zubin y Spring (1977), que considera a dicha vulnerabilidad como un rasgo permanente y a la esquizofrenia como un estado episódico- nos permite asumir que si todos los individuos (en mayor o menor grado) participamos del rasgo dimensional «vulnerabilidad a la esquizofrenia», será posible detectar marcadores de tal vulnerabilidad en aquellos individuos altamente propensos o de "alto riesgo" mucho antes de que se produzca el episodio esquizofrénico.

Así, pues, una vez que dispongamos de tales índices podremos identificar a las poblaciones de «alto riesgo». O lo que es lo mismo, al disponer de medidas de vulnerabilidad a la esquizofrenia, estaremos, consecuentemente, en condiciones de identificar grupos apropiados para participar en posibles programas de intervención preventiva. El paso siguiente será la elaboración de programas preventivos para impedir que tales individuos desemboquen en el episodio esquizofrénico.

Por tanto, llegados a este punto y dentro de la lógica establecida, las cuestiones relevantes a plantear serían las siguientes:

1) ¿Qué postula el modelo de vulnerabilidad?

2) ¿Qué marcadores de vulnerabilidad han sido identificados?

3) ¿Existe apoyo experimental de que tales índices estén relacionados con las poblaciones calificadas de "alto riesgo" en función de criterios diferentes? 
4) De ser así, ¿estamos preparados para detectar precozmente la esquizofrenia? Es decir, ¿para identificar individuos altamente vulnerables a la esquizofrenia antes de que se manifieste la psicosis?

5) Si la respuesta es afirmativa, ¿estamos preparados para prevenirla? $\mathrm{Y}$, si es así, ¿cómo?

A continuación trataremos de dar respuesta a las cinco cuestiones planteadas.

\section{EL MODELO DE VULNERABILIDAD}

Como hemos señalado antes, la etiología de la esquizofrenia sigue siendo un enigma aún no resuelto. En 1972, Zubin identificó seis modelos etiológicos (genético, del ambiente interno, neurofisiológico-anatómico, de la teoría del aprendizaje, evolutivo y ecológico) que se sitúan entre dos polos: el genético y el ecológico. Precisamente entre estos dos polos se ha dividido la opinión de los investigadores; encontrándonos con aquéllos que consideran a la esquizofrenia como un trastorno esencialmente biológico y aquéllos otros que la consideran como un trastorno básicamente psicosocial.

Frente a tal estado de cosas, podemos decir que han surgido dos alternativas. Por un lado, la propuesta de un modelo multifactorial y, por otro, la propuesta del modelo de vulnerabilidad. Hay que tener en cuenta, no obstante, que ambas posturas no son incompatibles. En un trabajo muy reciente, Ciompi (1984) considera la plausibilidad de un modelo multifactorial en el que podrían contribuir a la génesis de la esquizofrenia los siguientes factores biológicos y psicosociales: anormalidades constitucionales genéticamente determinadas, daño cerebral pre y perinatal, perturbación temprana de las relaciones madre-hijo, pautas de comunicación familiar ambiguas y contradictorias, e influencias sociales y culturales específicas. «En combinaciones variables, tales factores podrían llevar, eventualmente, a través de un círculo vicioso, a resultados bastante similares, y en particular a un déficit en el procesamiento de la información» (Ciompi, 1984, p. 638).

Por su parte, Zubin y Spring (1977) revisaron los seis modelos mencionados y comprobaron que existe un factor común a todos ellos que los conecta entre sí. Ese denominador común es un modelo de segundo orden al que llamaron "modelo de vulnerabilidad a la esquizofrenia".

El concepto de vulnerabilidad o propensión a la esquizofrenia constituye una constante implícita en gran parte de los seis modelos etiológicos ya mencionados. Sin embargo, en opinión de Zubin y Spring (1977), la contribución de cada uno de ellos a la comprensión de la etiología de la esquizofrenia es sólo parcial. Ninguno de tales modelos, a juicio de estos autores, hà sido puesto en cuestión por los datos experimentales aportados por los demás, lo que pone de manifiesto su compatibilidad y complementariedad. La estrategia de Zubin y Spring, de cara a plantear un modelo alternativo, se apoya en este hallazgo y es de naturaleza esencialmente pragmática. En efecto, tras analizarlos extrajeron un factor común -la vulnerabilidad- que permitía guiar la investigación en el marco de cada uno de los enfoques de la esquizofrenia. En este sentido, el concepto de vulnerabilidad parece ser —en opinión de Zubin (1978) - el candidato idóneo para 
explicar mucha de la aparente confusión y variabilidad de la investigación sobre la esquizofrenia.

Este modelo, que desde su publicación inicial en 1977 ha recibido sucesivas matizaciones (e.g., Spring y Zubin, 1978; Zubin y Steinhauer, 1981; Zubin, Magaziner y Steinhauer, 1983, y Nuechterlein y Dawson, 1984) postula que todo individuo participa de un determinado grado de vulnerabilidad a la esquizofrenia que, bajo determinadas circunstancias (agentes «estresógenos») se traducirá en un episodio esquizofrénico. En palabras de $\mathrm{Zu}$ bin (1978, p. 641), por vulnerabilidad se entiende "la predisposición a desarrollar un episido esquizofrénico cuando las circunstancias vitales (tanto externas como internas) producen el suficiente grado de estrés que supera (el umbral) la tolerancia al estrés de una persona" (ver fig. 1).

Lo realmente nuevo e importante que introducen Zubin y Spring (1977) es la distinción entre vulnerabilidad a la esquizofrenia, entendida como un rasgo relativamente permanente y duradero, y episodios del desorden esquizofrénico, considerados como estados que aparecen y desaparecen y que poseen, por tanto, un carácter temporal.

FIGURA 1

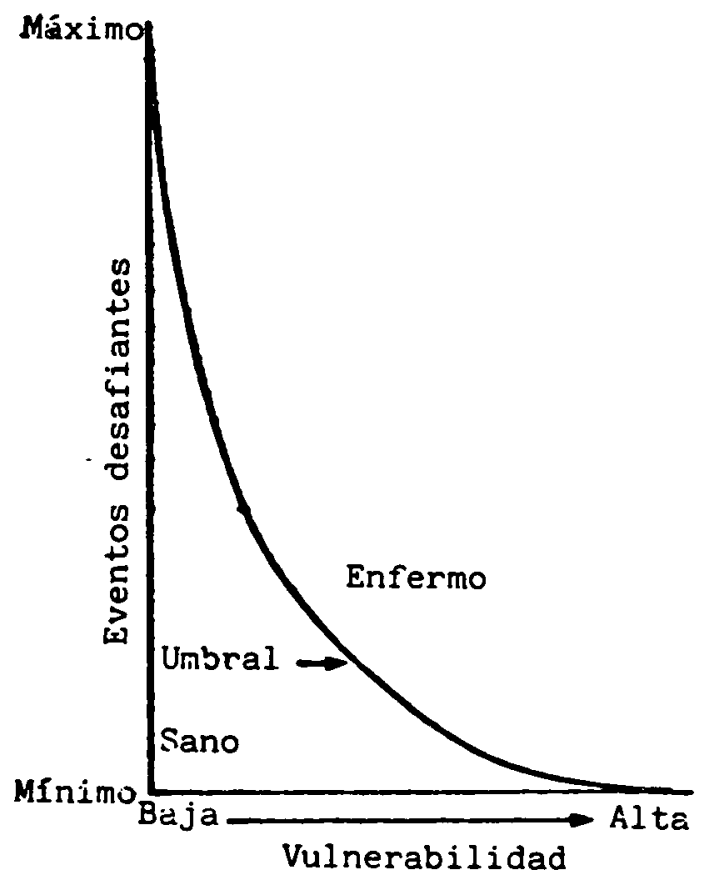

Relación entre vulnerabilidad y circunstancias vitales desafiantes. La ecuación para esta curva bipotética es $V S=K$, donde $V$ es el grado de vulnerabilidad, $S$ es el grado de estrés inducido por el evento desafiante y $K$ es una constante. (Zubin y Steinbauer, 1981).

Este modelo asume, pues, que el concepto de esquizofrenia no implica un desorden crónico, sino una vulnerabilidad permanente a desarrollar tal desorden. En este sentido «la esquizofrenia se convierte en una enfermedad episódica en el mismo sentido que la depresión, la epilepsia o la alergia» (Zubin, Magaziner y Steinhauer, 1983, p. 557). Recientes estudios lon- 
gitudinales, a los que ya hemos hecho referencia, han puesto de manifiesto que la esquizofrenia implica breves episodios de enfermedad, recuperación, recaída, nueva recuperación, etc.; lo que apoyaría la hipótesis de que lo que es relativamente permanente en la esquizofrenia es ese rasgo de vulnerabilidad y no los episodios o estados psicóticos.

El modelo se completa planteando:

a) El problema de la evaluación del umbral de vulnerabilidad que se concreta en la búsqueda de marcadores que permiten identificar individuos altamente vulnerables.

b) El papel que juegan los factores estresantes, enfrentándose con la dicotomía "formativo versus precipitante".

c) El rol de las variables moderadoras (las redes sociales, el nicho físico-ecológico y la personalidad premórbida) que serían las que previenen el episodio o, por el contrario, permiten su desarrollo.

Siguiendo con la línea planteada en nuestra introducción, sólo nos detendremos por razones pertinentes, en el apartado relativo a los marcadores de vulnerabilidad. Los apartados b) y c) serán por tanto, obviados.

\section{MARCADORES DE VULNERABILIDAD}

Ya hemos comentado que la vulnerabilidad es un rasgo relativamente permanente que predispone a la psicosis. Ahora bien, lo que no hemos dicho es que incluye componentes tanto genéticos como adquiridos. Así, pues, la vulnerabilidad debe ser considerada como un rasgo fenotípico que puede ser medio.

La naturaleza fenotípica de la vulnerabilidad y su categoría de rasgo es lo que permitirá el descubrimiento de los posibles marcadores. Enteniendo por tales aquellas características persistentes que pueden identificarse antes del primer comienzo de la esquizofrenia, que continúan concomitantemente con los síntomas y que perduran tras la remisión de éstos. En consecuencia, todo marcador de vulnerabilidad debe reunir las siguientes condiciones:

1) Poder ser identicado antes del primer episodio.

2) Continuar con la sintomatología.

3) Permanecer durante la remisión.

4) Encontrarse, con una probabilidad significativamente mayor, en los hermanos e hijos de los esquizofrénicos que en los pacientes psiquiátricos no-esquizofrénicos y en los controles sanos.

La puesta en práctica de esta estrategia ha seguido, básicamente, dos caminos: 1) la detección de sujetos de "alto riesgo" a la esquizofrenia que se concreta en el estudio de hipotéticos marcadores de vulnerabilidad en niños y/o adolescentes (parientes de esquizofrénicos) libres de toda sintomatología esquizofrénica, y 2) el estudio de marcadores de vulnerabilidad en esquizofrénicos adultos, remitidos o no, y que, por tanto, tienen un alto riesgo de episodios futuros.

Por razones absolutamente obvias en el contexto en que nos encontramos, nos detendremos sólo en la primera vía de investigación; esto es, la 
detección de marcadores de vulnerabilidad en niños de «alto riesgo». Esta línea de investigación, que tiene sus antecedentes en la década de los sesenta, se ha configurado en los últimos años como un instrumento de investigación especialmente útil por diferentes razones. Por ejemplo, podría ser la vía adecuada para resolver el problema metodológico que plantea la interpretación de las diferencias encontradas entre esquizofrénicos y normales, en el sentido de que aún no ha sido posible determinar inequívocamente si tales diferencias son la causa o el resultado de la esquizofrenia. Asimismo, al proporcionar información sobre las contingencias que desencadenan los episodios, permitiría el estudio más directo de la génesis y el desarrollo de la esquizofrenia. Por último, permitiría examinar los posibles programas preventivos de la esquizofrenia.

\section{ESTUDIOS CON POBLACIONES DE ALTO RIESGO}

Ante todo conviene precisar al máximo dos cuestiones que resultan ser de la máxima relevancia en este campo de estudio. Se trata de precisar los criterios para la selección de las muestras y la naturaleza de los marcadores estudiados.

\section{Selección de las muestras}

En su excelente revisión de 1974, Garmezy señala que en los estudios con niños de «alto riesgo» se han utilizado cuatro modelos conceptuales como criterios de selección de muestras.

El lugar predominante lo ha ocupado, y lo sigue ocupando (aunque después haremos algunas matizaciones al respecto), el modelo genético. Partiendo de la hipótesis de que la etiología de la esquizofrenia es genética, la mayor parte de estos estudios han utilizado como muestras niños con uno o dos progenitores esquizofrénicos. El primer estudio que siguió este modelo fue el de Mednick y Schulsinger (1968) con niños daneses. El rigor metodológico y los resultados obtenidos tuvieron tal impacto sobre la comunidad científica que, el hecho de que este modelo siga siendo el predomiante actualmente, obedece básicamente a esta razón. No obstante, otros investigadores, teniendo presentes los datos aportados por los estudios genéticos, han adoptado otros criterios. En concreto, los estudios genéticos han venido a demostrar que entre el $85-95 \%$ de los esquizofrénicos adultos no tienen padres esquizofrénicos y, posiblemente, entre el $60-70 \%$ de los esquizofrénicos no tengan ni parientes esquizofrénicos. Los estudios longitunales con niños de «alto riesgo" (genético) han comprobado, por su parte, que sólo un 10-16\% de ellos desarrollan después una esquizofrenia clínica.

¿Por qué, entonces, sigue siendo el modelo dominante en la actualidad? Porque los científicos han caído en la cuenta de que, en realidad, utilizar como muestra hijos de esquizofrénicos no constituye una prueba exclusiva de la contribución genética. El propio Mednick ha señalado recientemente (Mednick et al., 1982) que no es correcto igualar su estudio pionero con hijos de madres esquizofrénicas con un modelo genético de la esquizofre- 


\section{5}

nia. Como señala Keith (1982) el hecho de tener un progenitor esquizofrénico aumenta, en efecto, el riesgo de llegar a ser esquizofrénico; ahora bien, el que esa asociación sea ambiental o genética es algo que continúa abierto a la especulación. Sin olvidar que los genes, posiblemente, jueguen un papel importante, hay que tener en cuenta, asimismo, el impacto de un progenitor esquizofrénico como una influencia ambiental.

Además el modelo genético, Garmezy (1974) analiza otras tres alternativas, representadas por el modelo psicogénico el modelo sociogénico y el modelo del abandono precoz (que implicaría defectos durante el embarazo, el nacimiento y nutricionales). Como entedemos que ninguno de ellos presenta problemas especiales, vamos a pasar a señalar los procedimientos de selección de muestras utilizados en los estudios de «alto riesgo" que analizaremos después. Como vamos a comprobar, los tres criterios utilizados son perfectamente compatibles con los modelos más representativos señalados por Garmezy. En concreto, se trata de seleccionar:

1) Los parientes en primer grado de los pacientes esquizofrénicos, generalmente los hijos.

2) Aquellos individuos que muestran características de personalidad que se cree que están relacionadas con un riesgo elevado de desorden esquizofrénico (Steronko y Wood, 1978, y Chapman, Edell y Chapman, 1980).

3) Sujetos con características bioquímicas que han sido observadas en pacientes esquizofrénicos (Buchsbaum et al., 1978).

\section{Naturaleza de los marcadores}

Si aceptamos, como es nuestro caso, que la etiología de la esquizofrenia es multifactorial, los marcadores de vulnerabilidad a estudiar serían innumerables. Tal tarea podría realizarse y de hecho se está llevando a cabo por diferentes grupos de investigadores. Ahora bien, que tratáramos de hacer aquí una exposición de todos ellos sobrepasaría con creces todas las posibles expectativas. Consecuentemente, nos limitaremos a un campo que, además de ser de máxima actualidad en la psicología es hasta el momento el que está ofreciendo los resultados más consistentes dentro del modelo de vulnerabilidad a la esquizofrenia. Se trata del enfoque del procesamiento de la información o, más concretamente, se trata de revisar los estudios experimentales más relevantes que están permitiendo la identificación de marcadores cognitivos de vulnerabilidad a la esquizofrenia en poblaciones de "alto riesgo". Y para que la elección quede libre de toda sospecha de parcialidad por nuestra parte, expondremos la opinión al respecto que, hace sólo unos pocos meses, expresaba el prestigioso psiquiatra suizo Luc Ciompi (1984): «En un sentido amplio, las dificultades en el procesamiento de la información representan el denominador común más plausible de la vulnerabilidad premórbida de aquéllos que más tarde llegarán a ser esquizofrénicos" (p. 638; el subrayado es nuestro). 


\section{PROCESAMIENTO DE LA INFORMACION EN POBLACIONES DE ALTO RIESGO}

A continuación vamos a revisar una serie de estudios que han utilizado tareas de laboratorio con los tres tipos de poblaciones de "alto riesgo" antes mencionadas. Con el fin de facilitar una integración de los hallazgos, los distintos resultados de los tres tipos de poblaciones serán revisados juntos. Es importante subrayar que las medidas utilizadas cuentan con abundante evidencia experimental de que distinguen a los esquizofrénicos adultos (remitidos o no) de los sujetos de control.

\section{Amplitud de aprehensión de elección forzosa}

Se trata de una tarea de amplitud de aprehensión de informe parcial ideada por Estes y Taylor (1964). Puesto que no exige al sujeto que informe de todas las letras expuestas, esta tarea puede estimar adecuadamente la eficacia de la búsqueda visual en la memoria sensorial (icónica).

Asarnow, Steffy, McCrimmon y Gleghorn (1977, 1978), McCrimmon, Cleghron, Asarnow y Steffy $(1980)$, y Asarnow $(1979,1983)$ han comprobado que las muestras de niños de «alto riesgo» son deficientes en esta prueba con respecto a los niños de los grupos de control. Concretamente, en el primer estudio (Asarnow et al., 1978) utilizaron una muestra de niños de edades entre doce y dieciséis años, hijos de madres esquizofrénicas pero que habían sido adoptados, a una edad muy temprana por padres sanos. Como grupos de control utilizaron uno formado por niños adoptados por padres sanos e hijos biológicos de padres también sanos, y otro formado por niños no adoptados y con padres sanos. Las edades de los controles oscilaban igualmente entre doce y dieciséis años.

Los tres grupos fueron evaluados en una batería de ocho pruebas de atención, entre ellas la del «informe parcial de elección forzosa». Los resultados pusieron de manifiesto que la ejecución del grupo de «alto riesgo» era significativamente inferior a la de los dos grupos de control, sobre todo cuando se manipulaba la variable independiente «número de letras irrelevantes".

Más recientemente, Asarnow, Nuechterlein y Marder (1983) realizaron un estudio en el que el rendimiento en «amplitud de aprehensión» fue considerado como variable independiente. En concreto, comprobaron que un grupo de jóvenes sin historia de trastornos psiquiátricos, pero que habían tenido un rendimiento claramente bajo en la tarea de amplitud, alcanzaron puntuaciones significativamente más altas en la escala «esquizofrenia» del MMPI, la escalas de «esquizoidia» (Golden y Meehl, 1979), la escala de «esquizofrenismo" (Nielsen y Petersen, 1976) y la escala de «ideación mágica» (Eckbad y Chapman, 1983) que el grupo de sujetos de control.

Estos dos grupos de datos, junto con los provenientes de estudios con esquizofrénicos adultos (e.g., Asarnow y McCrimmon, 1978, 1981, 1982) sugieren que la eficacia de los procesos de búsqueda visual temprana, en presencia de distractores múltiples, puede estar alterada en las poblaciones con riesgo de esquizofrenia. Al mismo tiempo, los datos aportan evidencia en favor de la tarea "amplitud de aprehensión de elección forzosa" como un buen marcador de vulnerabilidad a la esquizofrenia. 
Tiempo de reacción: efecto de cruce

Diferentes estudios, en los que se ha utilizado el paradigma de tiempo de reacción (TR) simple variando los intervalos preparatorios, han comprobado que, mientras los sujetos normales son más rápidos (como es de esperar) con intervalos regulares, los esquizofrénicos sólo se comportan así con intervalos preparatorios (IPs) muy cortos (inferiores a los dos segundos). Con intervalos largos los esquizofrénicos son más lentos con IPs regulares que con IPs irregulares. Esta inversión es conocida como «efecto de cruce" y ha sido observada en esquizofrénicos internados (e.g., Rodnick y Shakow, 1940; Bellisimo y Steffy, 1972, 1075; De Amicis y Cromwell, 1979, y Steffy y Galbraith, 1980) y en esquizofrénicos remitidos (Strauss et al., 1979).

Marcus (1973) aplicó este paradigma a veinte hijos de madres esquizofrénicas y a diferentes grupos de control. El grupo de «alto riesgo» mostró un patrón de resultados distintos al de los otros grupos pero sólo en cuanto a su mayor lentitud en TR; sin embargo, no mostraron el patrón típico del «efecto de cruce».

Este mismo o similar cuadro de resultados ha sido encontrado en otros cuatro trabajos (Van Dyke et al., 1975; Asarnow et al., 1977, 1978, y Spring, 1980) con sujetos de "alto riesgo" lo que sugiere cierta inconsistencia del «efecto de cruce» como marcador de vulnerabilidad. No obstante, y por eso hablábamos de cierta inconsistencia, hay dos trabajos que han encontrado el patrón típico esquizofrénico del «cruce» en muestras de alto riesgo. Por un lado, De Amicis y Cromwell (1979) encontraron que un grupo de parientes no psicóticos de esquizofrénicos presentaban un significativo «efecto de cruce» cuya magnitud estaba en función del grado de parentesco (primer o segundo grado) con los pacientes esquizofrénicos. Por otro Simons, Macmillan y Ireland (1982) comprobaron que un grupo de estudiantes, que habían puntuado alto en las escalas de «aberración perceptual», y "anhedonia física», mostraban evidencia del «efecto de cruce» con respecto a otro grupo de estudiantes que habían puntuado bajo en las dos escalas de propensión a la psicosis.

\section{Atención selectiva}

En el estudio citado de Asarnow et al. (1978) se incluía una tarea que consistia en escribir una serie de dígitos aleatorios que eran presentados binauralmente, al tiempo que ignoraban otra serie de dígitos que se presentaba sólo por un oído. Los hijos de madres esquizofrénicas mostraron una tendencia significativa a cometer más errores que los niños de los dos grupos de control.

Orvaschel et al. (1979), sin embargo, no encontraron ninguna alteración significativa, en una tarea de escucha dicótica, en los hijos de madres esquizofrénicas comprados con los hijos de padres normales. No obstante, este estudio difirió del anterior en que sólo presentaban tres dígitos por cada oído, lo que no implicaba seleccionar un mensaje monoaural frente a otro binaural. 
Recientemente, Spring et al. (1983) han presentado datos que suponen la prueba más fiable para un déficit en escucha dicótica en los parientes en primer grado de los pacientes esquizofrénicos. Utilizando una tarea de sombreado, comprobaron que los parientes en primer grado (padres e hijos) de los pacientes esquizofrénicos cometían significativamente más errores de intrusión del mensaje irrelevante que los sujetos de control normales.

Si bien estos estudios parecen apoyar la hipótesis de una alteración de la atención selectiva en personas de "alto riesgo" en tareas que sólo implican el proceso de "filtraje» otros estudios no han encontrado tal déficit selectivo de una manera consistente, cuando el proceso implicado era el «pigeon-holing» (Broadbent, 1971).

\section{Atención sostenida o vigilancia: El test de ejecución continua (CPT)}

Disponemos de abundante evidencia experimental de que el CPT discrimina la ejecución de los pacientes esquizofrénicos y de los esquizofrénicos remitidos de la de los grupos de control (e.g., Wohlberg y Kornetsky, 1973; Kornetsky y Orzack, 1978, y Asarnow y McCrimmon, 1978). Tanto los esquizofrénicos como los esquizofrénicos remitidos cometen significativamente más errores de omisión y de comisión que los grupos de control.

En los estudios con poblaciones de "alto riesgo" podemos establecer dos grupos de estudios. Por un lado, tendríamos aquellos trabajos que han utilizado un CPT simple y que, por tanto, implicaban una carga baja del procesamiento; y por otro, aquellos que han utilizado un CPT que implicaba una carga mayor del procesamiento. Los primeros, entre los que destacaríamos los trabajos de Asarnow et al. (1978), Nuechterlein (1983), Cornblatt y Erlenmeyer-Kimling (1984), no han encontrado diferencias significativas entre los grupos de "alto riesgo" y los diferentes grupos de comparación. Los segundos, entre los que se encuentran los trabajos de Buchsbaum et al. (1978), Erlenmeyer-Kimpling y Cornblatt (1978), Nuechterlein (1984), Nuechterlein, Edell y West (en preparación) y Nuechterlein, Asarnow y Marder (en preparación), sí han obtenido resultados positivos al comparar los grupos de "alto riesgo" con los distintos grupos de control. Así pues, cuando la tarea de vigilancia (operativizada con el CPT) implica una tarea que requiere una carga del procesamiento relativamente alta, los grupos de «alto riesgo» muestran un funcionamiento cognitivo alterado que puede ser considerado como un marcador consistente de vulnerabilidad a la esquizofrenia.

\section{Otros estudios}

La investigación en este campo se ha extendido también a otros aspectos del procesamiento de la información. Concretamente a los procesos de reconocimiento y recuerdo en la memoria a corto plazo, a la memoria semántica, a la formación de conceptos, etc. Los resultados, sin embargo, no presentan suficiente evidencia, en ningún sentido, que permita establecer diferencias significativas entre la población de «alto riesgo» y los grupos de comparación. 


\section{Conclusiones}

A la luz de los datos expuestos, y teniendo en cuenta algunas de las preguntas planteadas al comienzo de este trabajo, podríamos hacer las siguientes consideraciones:

1. Disponemos de algunos marcadores de vulnerabilidad a la esquizofrenia, en la esfera del procesamiento de la información que, junto con marcadores de otra naturaleza, podrían ir configurando esa batería tan esperada que permitiera la identificación precoz de los sujetos que podrían convertirse en esquizofrénicos.

2. Existe algún apoyo experimental - aunque todavía escaso- de que los marcadores cognitivos guardan una estrecha relación con marcadores de distinta naturaleza (vg. bioquímica, genética, familiar, etc.).

3. De todas formas, aún es pronto para poder afirmar que disponemos de un instrumento válido y fiable que permita la detección precoz de la esquizofrenia. No obstante, hay que ser optimistas, porque estamos convencidos de encontrarnos en el camino correcto. Prueba de ello son algunos de los resultados — aún sin publicar- sobre la validez de los predictores cognitivos analizados como consecuencia de los diferentes estudios longitudinales todavía en marcha.

4. El no haber alcanzado aún el nivel óptimo no implica la inviabilidad de los programas de intervención preventiva.

5. El conocimiento disponible permite pensar, sobre un fundamento empírico, que la esquizofrenia tiene su génesis en los primeros años de la vida.

6. Finalmente, los programas preventivos serán tanto más eficaces cuanto más precozmente se apliquen.

Sobre las consideraciones 4, 5 y 6 disponemos de algunos datos. Somos conscientes de que son escasos, pero al mismo tiempo sugerentes.

Así pues, analicemos, antes de finalizar este trabajo, los aspectos más relevantes de la experiencia disponible sobre los programas preventivos.

\section{ALGUNOS INTENTOS ESPECIFICOS PARA PREVENIR LA ESQUIZOFRENIA}

En realidad, los programas preventivos de la esquizofrenia se han reducido hasta ahora - salvo alguna excepción- a informes piloto de investigadores que, de un modo un tanto informal, han intentado algún tipo de intervención preventiva.

A pesar de la importancia que estamos atribuyendo al procesamiento de la información y a los marcadores cognitivos de vulnerabilidad, todavía no se ha puesto en marcha ningún programa preventivo partiendo de tales presupuestos. A lo sumo, se han hecho algunas especulaciones teóricas sobre qué dirección o qué objetivos debieran cubrir tales programas. Ahora bien, esto no supone una crítica negativa a los investigadores de tal enfoque. A la vista de los datos revisados, consideramos que su actitud es, por encima de todo, coherente y de una envidiable conciencia científica. Un programa preventivo, si quiere ser eficaz tiene, necesariamente, que partir de una só- 
lida fundamentación teórica y empírica. Mientras no se disponga de ésta, la espera y la prudencia serán las recomendaciones más pertinentes. Hasta ahora, como decíamos, sólo disponemos de algunos informes piloto, como por ejemplo el de Fish (1982) y el de Watt et al. (1982), en los que se ha puesto de relieve que el voluntarismo, aparte de ser muy respetable, suele aportar bastante poco a la ciencia y a la comunidad. No obstante, sus "programas" de seguimiento -el de Fish de veinte-veintisiete años, y el de Watt et al., de siete meses (aunque continúa en la actualidad) - nos han ofrecido algunos datos que pudieran resultar valiosos, siempre y cuando se utilicen con la precaución con la que se debe tratar todo dato escasamente verificado.

Resumiendo, consideramos que la mayor debilidad de estos dos programas se encuentra en los criterios utilizados para definir las poblaciones apropiadas para la intervención preventiva. En el caso de Fish se utilizó como criterio de medida lo que ella llamó «retraso evolutivo global» (pandevelopmental retardation, $P R D$ ). Un indice establecido en 1952, de los desórdenes neurointegradores, que la propia autora considera completamente trasnochado (Fish, 1982). En el caso de Watt et al., el instrumento fue, básicamente, un registro de la conducta del niño en el aula en base a cuatro factores: motivación escolar, extraversión, armonía y estabilidad emocional. Si bien este instrumento permite clasificar a los alumnos, sobre todo en cuanto a su vulnerabilidad emocional, su relación con la esquizofrenia no parece muy fiable a la luz de los resultados obtenidos en su programa preventivo. Quizá sería más oportuno considerarlo como un criterio para evaluar la vulnerabilidad a trastornos psicopatológicos en general.

Y como prueba de la falta de consistencia de estos programas, valga como muestra el hecho de que mientras Fish considera que la edad adecuada para la aplicación de los programas preventivos no debe superar los 3 años - concretamente llega a decir que a "la edad de 3-6 años es ya demasiado tarde para prevenir las alteraciones cognitivas y socio-afectivas" (Fish, 1982, p. 236)-, para el grupo de Watt, los programas deben aplicarse cuando los chicos se encuentran en la «escuela secundaria».

De tales trabajos, no obstante, también podemos extraer alguna aportación de valor. Desde nuestro punto de vista, estos dos programas han puesto de manifiesto explicitamente algo que viene a apoyar claramente la tesis que aquí estamos manteniendo. A saber, la necesidad de recurrir a pruebas refinadas de laboratorio para la identificación de individuos vulnerables a la esquizofrenia. Entendemos que no es necesario recordar que los índices revisados en apartados anteriores se ajustarían a tal necesidad.

No quisiéramos acabar esta comunicación sin hacer mención al que, hasta el momento, puede considerarse como el único programa real de prevención de la esquizofrenia. Se trata del Proyecto Mauricio, puesto en marcha por Mednick y su equipo de investigación en 1972, y cuyos resultados (aún parciales) han aparecido en al menos dos trabajos (Mednick et al., 1982, 1984).

Dicho proyecto tiene su origen en el estudio longitudinal de alto riesgo con niños daneses iniciado en 1962 y terminado en 1967 (Mednick y Schulsinger, 1968). En aquel trabajo, Mednick y Schulsinger llevaron a cabo un seguimiento de 207 niños de alto riesgo a la esquizofrenia (todos ellos hijos de mujeres esquizofrénicas) y de 104 niños controles (hijos de mujeres nor- 


\section{1}

males). En 1967, tras cinco años de seguimiento, estos investigadores comprobaron que veinte de los niños de alto riesgo habían desarrollado distintos trastornos psiquiátricos (no necesariamente esquizofrenia). Un análisis detallado puso de manifiesto que el mejor discriminador de los sujetos con trastornos era una variable del sistema nervioso autónomo (la tasa rápida de recuperación de la conductancia de la piel). Posteriormente, Mednick et al. (1978) demostraron que las variables del sistema nervioso autónomo son buenos predictores de esquizofrenia.

De acuerdo con su plan general de investigación, el siguiente paso supondría examinar «una población normal con nuestros tests a fin de detectar aquellos individuos que son esquizofrénicos potenciales y explorar, a continuación, la posibilidad de intervención" (Mednick, 1960; citado en Mednick et al., 1982). Cuando los autores se encontraban planificando ese proyecto para ponerlo en marcha en Dinamarca, la Organización Mundial de la Salud les ofreció la oportunidad y el apoyo financiero para realizarlo en la isla de Mauricio.

En Mauricio, los autores comenzaron evaluando psicofisiológicamente (SNA-conductancia de la piel) a 1.800 niños de 3 años de edad. Además, observaron y codificaron la conducta de juego, el desarrollo cognitivo, el electroencefalograma, una entrevista familiar, un examen médico general e información perinatal de los 1.800 niños. En contraste con el trabajo pionero danés, donde la selección de los niños de alto riesgo se había hecho sobre la base de características paternas conocidas, los sujetos de alto riesgo de Mauricio fueron seleccionados tomando como base de datos psicofisiológicos. Era, pues, la primera vez que se realizaba un examen de una población, con el objetivo de obtener una detección precoz de niños de alto riesgo, con técnicas psicofisiológicas.

De la población inicial (1.800 niños) fueron identificados 200 niños de alto riesgo por presentar alteraciones en la conductancia de la piel similares a las del grupo danés. El programa de intervención se inició en 1973, cuando 100 de tales niños fueron enviados a dos guarderías (junto con niños de bajo riesgo), al tiempo que los 100 restantes permanecieron en la comunidad (controles de la comunidad). En las guarderías no se llevó a cabo ningún programa específico de intervención. No obstante, las guarderías presentaban ciertas características distintivas, a saber: a) los 100 niños procedian, casi en su totalidad, de familias pobres; los niños eran llevados y traídos en autobús, en la guardería permanecían desde las 9 a.m. hasta las 3 p.m., y allí se les daba una comida caliente que, con toda seguridad, tenía un contenido proteico más alto que la que recibían los controles de la comunidad. b) En base a los procedimientos de selección, la guargería tenía una densidad muy alta de niños autonómicamente sensibles (alrededor del $50 \%$ ). c) Los directores y los maestros estaban muy motivados y bien entrenados; no obstante, en ningún momento nadie fue informado en Mauricio sobre el estatus autonómico de los niños.

En diciembre de 1976, justo antes de que comenzaran la escuela primaria, todos los niños (de alto y bajo riesgo) fueron evaluados conductualmente a fin de compararlos y de estimar el efecto de la guardería. La evaluación se efectuó utilizando tres criterios: mirar (el observador registraba el tiempo que el niño estaba inactivo y mirando a otros), interacción positiva (registro del tiempo que el niño hablaba o jugaba cooperativamente 
con los otros niños) y juego constructivo en solitario (registro del tiempo que el niño permanecía solo jugando constructivamente con juguetes). El tiempo de observación total para cada niño fue de ocho minutos.

Comparando los resultados de los grupos de alto y bajo riesgo de las guarderías y de la comunidad, se observó que: 1) tanto los grupos de alto como de bajo riesgo redujeron sustancialmente su «mirar inactivo» después de haber asistido a las guarderías; 2) los niños de bajo riesgo de la comunidad presentaban una conducta relativamente alta de «interacciones positivas» y «mirar" pero, tras la experiencia de la guardería, redujeron esas actividades en beneficio de más «juego constructivo»; 3) los niños de alto riesgo de la comunidad tenían índices altos en «juego constructivo», y 4) el mayor efecto de la experiencia de las guarderías para los niños de alto riesgo fue su incremento en las «interacciones positivas» de naturaleza social.

Las interpretaciones que se han dado en estos resultados (Mednick $e t$ al., 1982, 1984) son las siguientes: 1) los criterios de selección psicofisiológica correlacionaban con conductas específicas antes de la influencia de la guardería. 2) La guardería afecta a la conducta social y de juego de los niños. 3) Los efectos diferenciales de la experiencia de la guardería sobre la conducta de los niños está en función de las características psicofisiológicas utilizadas en la selección de las muestras.

Desde 1976, el programa continúa con los niños en las escuelas primarias. Sin embargo, aún no se han publicado datos relativos a dicho período. Como señalan los autores (Mednick et al., 1982, 1984), lo más alentador de este Proyecto es el hecho de que el mayor cambio observado se dio en el grupo de alto riesgo de guarderías al mostrar un sorprendente incremento -incluso mayor que el de los niños de bajo riesgo- de las «interacciones positivas" de clara naturaleza social. Evidentemente, y así lo señalan Mednick et al. $(1982,1984)$, el éxito práctico de este proyecto dependerá de que sea correcta la suposición de que la variable del SNA elegida predispone a la esquizofrenia; es decir, que es un buen marcador de vulnerabilidad a la esquizofrenia.

Digamos, para terminar, que es posible que algunas o muchas de las cuestiones planteadas no hayan recibido respuesta satisfactoria por nuestra parte, pero los datos disponibles no permiten ir más allá. Una cosa son los deseos y otra muy distinta la realidad. Todos deseamos que la prevención de la esquizofrenia sea un hecho, pero la realidad nos dice que aún no estamos preparados. No obstante, el panorama no debe ser evaluado con pesimismo. Consideramos que si en sólo dos décadas se han alcanzado unos objetivos tan importantes como los comentados a lo largo de este trabajo, todas las personas que, de una u otra forma, estamos implicados en la esquizofrenia, tenemos la obligación de manifestar un sentimiento de optimismo.

\section{Referencias}

ASARNOW, R. F. (1979). Behavioral and laboratory markers of risk for shizophrenia in adolescents. Comunicación presentada en el Meeting of the American Psychological Association.

ASARNOW, R. F. (1983). Schizophrenia. En R. E. Tarter (comp.): The child at psychiatric risk. Oxford: Oxford University Press. 
ASARNOW, R. F., y MCCRIMMON, D. R. (1978). Residual performance deficit in clinically remitted schizophrenics: A marker of schizophrenia? J. of Abnormal Psychology, 87, 597-608.

ASARNOW, R. F., y MCCRImmon, D. J. (1981). Span of apprehension deficits during postpsychotic stages of schizophrenia. Archives of General Psychiatry, 38, 1006-1011.

ASARNOW, R. F., y MCCRIMMON, D. J. (1982). Attention/information processing, neuropsychological functioning, and thougt disorder during the acute and partial recovery phases of schizophrenia: A longitudinal study. Psychiatry Research, 7, 309-319.

ASARNOW, R. F., NuECHTERLEIN, K. H., y MARDER, S. R. (1983). Span of apprehension performance, neuropsychological functioning and indices of psychosis-proneness. $J$. of Nervous and Mental Diseases, $171,662-669$.

Asarnow, R. F.; Steffy, R. A.; MCCRimmon, D. J., y Cleghorn, J. M. (1977). An attentional assessment of foster children at risk for schizophrenia. J. of Abnormal Psychology, $86,267-275$.

Asarnow, R. F.; Steffy, R. A.; MCCrimmon, D. J., y Cleghorn, J. M. (1978). An attentional assessment of foster children at risk for schizphrenia. En L. C. Wynne, R. L. Cromwell y S. Matthysse (comps.): The nature of schizopbrenia: New approaches to research and treatment. Nueva York: John Wiley and Sons (pp. 339-358).

BELlissimo, A., y STEFFY, R. A. (1972). Redundancy-associated deficit in schizophrenic reaction time performance. J. of Abnormal Psychology, 80, 299-307.

BELlissimo, A., y STEFFY, R. A. (1975). Contextual influences on crossover in the reaction time performance of schizophrenics. J. of Abnormal Psychology, 84, 210-220.

BLEULER, M. (1978). The schizophrenic disorders: Lonf-term patient and family studies. Nueva York: Yale University Press.

BRoAdBent, D. E. (1971). Decision and stress. Londres: Academic Press, Inc.

Buchsbaum, M. S.; Murphy, D. L.; Coursey, R. D.; LAKe, C. R., y Ziegler, M. G. (1978). Platelet monoamine oxidase, plasma dopamine-beta-hydroxylase and attention in a wiochemical high risk" sample. J. of Psychiatric Research, 14, 215-224.

Chapman, L. J.; Edell, W. S., y Chapman, J. P. (1980). Physical anhedonia, perceptual aberration and psychosis proneness. Schizophrenia Bulletin, 6, 639-653.

CiompI, L. (1980). The natural history of schizophrenia in the long term. British J. of Psychiatry, 136, 413-420.

CIOMPI, L. (1984). Is there really a schizophrenia? The long-term course of psychotic phenomena. British J. of Psychiatry, 145, 636-640.

CORNBLATT, B., y ERLENMEYER-KIMLING, L. (1984). Early attentional predictors of adolescent behavioral disturbances in children at risk for schizophrenia. En N. F. Watt, E. J. Anthony, L. C. Wynne, y J. E. Rolf (comps.): Children at risk for schizopbrenia: A longitudinal perspective. Nueva York: Cambridge University Press.

DE AMICIS, L. A., y CROMWELL, R. L. (1979). Reaction time crossover in process schizophrenic patients, their relatives and control subjects. J. of Nervous and Mental Disease, 167, 593-600.

ERLENMEYER-KIMLING, L., y CORNBLATT, B. (1978). Attentional measures in a study of children at high-risk for schizophrenia. En L. C. Wynne, R. Cromwell y S. Matthysse (comps.): Nature of schizophrenia: New approaches to research and treatment. Nueva York: John Wiley and Sons (pp. 359-365).

Estes, W. K., y TAYLOR, H. A. (1964). A detection method and probavilistic models for asssessing informstion processing from brief visual displays. Proceeding of the National Academy of Sciences of the United States of America, 52, 446-454.

FISH, B. (1982). Attempts at intervention with high-risk children, from infancy on. En M. J. Goldstein (comp.): Preventive intervention in schizophrenia: Are we ready? DHHS Pub. No. (ADM) 82-1111. Washington, DC: Superintendent of Documents, US Governement Printing Office, pp. 226-241.

GARMEZY, N. (1974). Children at risk: The search for the antecedents of schizophrenia. Part II: Ongoing research programs, ussues and intervention. Schizopbrenia Bulletin, 1 , 55-125.

HubER, G.; Gross, G.; SCHUTTLER, R., y LiNz, M. (1980). Longitudinal studies of schizophrenic patients. Schizophrenia Bulletin, 6, 592-605.

KEITH, S. J. (1982). Broadening our concepts of high-risk research. En J. J. Goldstein (comp.): Preventive intervention in schizophrenia: Are we ready? DHHS Pub. No. (ADM) 82-1111. Washington DC: Superintendent of Documents, V. S. Government Printing Office, pp. 305-310.

KORNETSKY, C., y ORZACK, M. H. (1978). Physiological and behavioral correlates of attention dysfunction in schizophrenic patients. En L. C. Wynne, R. L. Cromwell y S. Matthysse (comps.): The nature of schizophrenia: New approaches to reseach and treatment (pp. 196-204). Nueva York: John Wiley and Sons.

MARCUS, L. M. (1973). Studies of attenttion in children vulnersable to psychopathology. Dissertation Abstracts International, 33, 5023-B (Univ. Microfilm, 73-10, 606).

MCCRImmon, D. J.; Cleghorn, J. M.; Asarnow, R. F., y STEFFy, R. A. (1980). Children at risk for schizophrenia: Clinical and attentional characteristics. Archives of General Psychiatry, 37, 671-674. 
MEDNICK, S. A., y SCHULSINGER, F. (1968). Some premorbid characteristics related to breakdown in children with schizophrenic mothers. J. of Psychiatric Research, 6, 354-362.

Mednick, S. A.; SChulsinger, F.; Teasdale, T. W.; SCHulsinger, H.; Venables, P. H., y ROCK, D. R. (1978). Schizophrenia in high-risk children: Sex differences in predisponing factors. En G. Serban (comp.): Cognitive defects of the development of mental illness. Nueva York: Bruner/Mazel.

Mednick, S. A.; Venables, P. H.; SChulsinger, F., y Cudeck, R. (1982). The Mauritius project: An experiment in primary prevention. En M. J. Goldstein (ed.): Preventive intervention in schizophrenia: Are we ready? DHHS Pub. (ADM) 82-1111. Washington, DC: Superintendent of Documents, US Government Printing Office, pp. 287-296.

MedNicK, S. A.; VAN DuSEN, K.; CUDECK, R., y SCHULSINGER, F. (1984). The Mauritius Project. En S. A. Mednick, M. Harway y K. M. Finello (comps.): Handbook of longitudinal research. Volume 1: Birth and childhood coborts (pp. 500-507). Nueva York: Praeger.

NUECHTEELEIN, K. H. (1983). Signal detection in vigilance tasks and behavioral attributes among offspring of schizophrenics mothers and among hyperactive children. $J$. of Abnormal Psychology, 92, 4-28.

NUECHTERLEIN, K. H. (1984). Sustained attention among children vulnerable to adult schizophrenia and among hyperactive children. En N. F. Watt, E. J. Anthony, L. C. Wynne y J. E. Rolf (comps.): Children at risk for schizophrenia: A longitunal perspective. Nueva York: Cambridge University Press.

NUECHTERLEIN, K. H., y DAwSON, M. E. (1984). A heuristic vulnerability/stress model of schizophrenic episodes. Schizophrenia Bulletin, 10, 300-312.

NueCHTERLEIN, K. H.; AsARnOW, R. F., y MARDER, S. (en preparación). Perceptual sensitivity during vigilance as an indicator of psychosis proneness.

NueChTERLEIN, K. H.; EDELL, W. S., y WEST, A. P. (en preparación). Signal detection during vigilance and span of apprehension in nonpsychotic schizotypal individuals.

ORVASCHEL, H.; MEDNICK, S.; SCHULSINGER, F., y ROCK, D. (1979). The children of psychiatrically disturbed parents: Differences a function of the sex of the sick parent. Archives of General Psychitry, 36, 691-695.

RODNICK, E., y SHAKOW, D. (1940). Set in the schizophrenic as measured by a composite reaction time index. American Journal of Psychiatry, 97, 214-225.

SHAPIRO, R., y SHADER, R. (1979). Selective review of previous follow-up studies of schizophrenia and other psychoses. En Schizophrenia: An Internatioval Follow-up Study. Nueva York: Wiley, pp. 11-43.

SimONS, R. F.; Macmillan, F. W., y IReland, F. B. (1982). Reaction-time crossover in preselected schizotypic subjects. J. of Abnormal Psychology, 91, 414-419.

SPRING, B. J. (1980). Shift of attention in schizophrenics, siblings of schizophrenics, and depressed patients. J. of Nervous and Mental Disease, 168, 133-140.

SPRING, B. J.; LEVITT, M.; BRIGGS, D., y BENET, M. (1983). Distractibility in relatives of schizophrenics. Comunicación presentada en 91st Annual Convention of the American Psychological Association. Anaheim, CA.

SPRING, B. J., y ZUBIN, J. (1978). Attention and information processing as indicators of vulnerability to schizophrenic episodes. J. of Psychiatric Research, 14, 289-302.

STEFFY, R: A., y GALBRATTH, K. (1980). Relation between latency and redundancy-associated deficit in shizophrenic reaction tinme performance. J. of Abnormal Psychology, 89, 419-427.

STERONKO, R. J., y WOODS, D. J. (1978). Impairment in early stages of visual information processing in nonpsychotic schizotypical individuals. J. of Abnornmal Psychology, 87, 481-490.

STrauss, M. E.; Bohannon, W. E.; Kaminsky, M. J., y Kharabi, F. (1979). Simple reaction time crossover in schizophrenic otupatients. Schizoprenia Bulletin, S, 612-615.

VAN DYKE, J. L.; ROSENTHAL, D., y RASMUSSEN, P. V. (1975). Effects of inheritance and rearing on reaction time. Canadian Journal of Bebavioral Science, 7, 223-236.

WATT, N. F.; GRUBb, T. W., y ERLENMEYER-KimLING, L. (1982). Social, emotional and intellectual behavior at school at high risk for schizophrenia. J. of Abnormal Psychology, 50, 171-181.

WOHLBERG, G. W., y KORNETSKY, C. (1973). Sustained attention in remitted schizophrenics. Archives of General Psychiatry, 28, 533-537.

ZubIN, J. (1972). Scientific models for psychopathology in the'70s. Seminar Psychiatry, 4, 283-296.

ZUBIN, J. (1978). Concluding comments. En L. C. Wynne, R. L. Cromwel! y S. Matthysse (eds.): The nature of schizophrenia. Nueva York: Wiley.

ZUBIN, J.; MAGAZINER, J., y STEINHAUER, S. R. (1983). The metaporphosis of schizoprenia: from chronicity to vulnerability. Psychological Medicine, 13, 551-571.

ZUBIN, J., y SPRING, B. (1977). Vulnerability: A new view of schizophrenia. J. of Abnormal Psychology, 86, 103=126.

ZubiN, J., y STEINHAUER, S. (1981). How to break the logjam in schizophrenia: A !ook beyond genetics. J. of Nervous and Mental Disease, 169, 477-492. 